\title{
Fractional Fourier transforms of hypercomplex signals
}

\author{
Hendrik De Bie • Nele De Schepper
}

Received: date / Accepted: date

\begin{abstract}
An overview is given to a new approach for obtaining generalized Fourier transforms in the context of hypercomplex analysis (or Clifford analysis). These transforms are applicable to higher-dimensional signals with several components and are different from the classical Fourier transform in that they mix the components of the signal.

Subsequently, attention is focused on the special case of the so-called Clifford-Fourier transform where recently a lot of progress has been made. A fractional version of this transform is introduced and a series expansion for its integral kernel is obtained. For the case of dimension 2, also an explicit expression for the kernel is given.
\end{abstract}

Keywords Hypercomplex analysis · fractional transform $\cdot$ generalized Fourier transform $\cdot$ CliffordFourier transform

\section{Introduction}

Recently, there has been an increasing interest in the theory of hypercomplex signals (i.e. functions taking values in a Clifford algebra, a generalization of the complex numbers) and the possibility of defining and using Fourier transforms (FTs) that interact with the Clifford algebra structure. This has been investigated from a practical engineering point of view (see e.g. [1-5]) but also from a purely mathematical point of view (see e.g.

H. De Bie, N. De Schepper

Department of Mathematical Analysis

Faculty of Engineering and Architecture

Ghent University

Galglaan 2, 9000 Ghent, Belgium

E-mail:

Hendrik.DeBie@UGent.be,

Nele.DeSchepper@UGent.be
[6-8]) using the function theory of Clifford analysis established in a.o. $[9,10]$. Also in applications, there is a lot of interest in having available a good hypercomplex Fourier transform (e.g. in GIS research, see [11]).

In the context of this paper, a hypercomplex signal is a function of $m$ real variables $x_{1}, \ldots, x_{m}$ taking values in a Clifford algebra $\mathcal{C} l_{0, m}$. This means that our signals can equivalently be represented by a vector

$\left[f_{1}\left(x_{1}, \ldots, x_{m}\right), \ldots, f_{2^{m}}\left(x_{1}, \ldots, x_{m}\right)\right]$

with at most $2^{m}$ components $f_{i}\left(x_{1}, \ldots, x_{m}\right)$ which are real-valued signals.

There are quite a few drawbacks to most of the kernels proposed so far in the literature. First, several authors work only in low dimensions (dimension 3 or 4 , enabling them to use quaternions instead of a full Clifford algebra) which is usually because they have a specific application in mind in these dimensions (e.g. in color image processing). Second, most authors use ad hoc formulations for the kernel function of their transforms, after which the properties of the related transform are studied in detail. A notable exception is the paper [12], where the authors give a general definition of a hypercomplex FT, encompassing many of the previous definitions.

In recent work (see [13-17]) we have developed a different methodology: we start from a list of properties or general mathematical principles we want a hypercomplex Fourier transform to have, and then determine all kernels that satisfy these properties.

In the present paper, after a short introduction to Clifford algebras and analysis, we give an overview of the results obtained so far. In particular we devote our attention to the so-called Clifford-Fourier transform (as studied in $[17,13]$ ). Subsequently, we introduce a new fractional version of the Clifford-Fourier transform 
(CFT). We determine a series expansion for its integral kernel in all dimensions. When restricting to the case of dimension 2, we are able to obtain an explicit expression for the kernel. In that special case, we can also derive further properties of the fractional CFT.

\section{Preliminaries}

Clifford analysis (see e.g. $[9,10]$ ) is a theory that offers a natural generalization of complex analysis to higher dimensions. To $\mathbb{R}^{m}$, the Euclidean space in $m$ dimensions, we first associate the Clifford algebra $\mathcal{C} l_{0, m}$, generated by the canonical basis $e_{i}, i=1, \ldots, m$. These generators satisfy the multiplication rules $e_{i} e_{j}+e_{j} e_{i}=-2 \delta_{i j}$.

The Clifford algebra $\mathcal{C} l_{0, m}$ can be decomposed as $\mathcal{C} l_{0, m}=\oplus_{k=0}^{m} \mathcal{C} l_{0, m}^{k}$ with $\mathcal{C} l_{0, m}^{k}$ the space of $k$-vectors defined by

$\mathcal{C} l_{0, m}^{k}:=\operatorname{span}\left\{e_{i_{1} \ldots i_{k}}=e_{i_{1}} \ldots e_{i_{k}}, i_{1}<\ldots<i_{k}\right\}$.

More precisely, we have that the space of 1 -vectors is given by $\mathcal{C} l_{0, m}^{1}=\operatorname{span}\left\{e_{i}, i=1, \ldots, m\right\}$, a space obviously isomorphic with $\mathbb{R}^{m}$. The space of so-called bivectors is given explicitly by $\mathcal{C} l_{0, m}^{2}=\operatorname{span}\left\{e_{i j}=e_{i} e_{j}, i<\right.$ $j\}$.

We identify the point $\left(x_{1}, \ldots, x_{m}\right)$ in $\mathbb{R}^{m}$ with the vector variable $\underline{x}$ given by $\underline{x}=\sum_{j=1}^{m} x_{j} e_{j}$. The Clifford product of two vectors splits into a scalar part and a bivector part:

$\underline{x} \underline{y}=\underline{x} \cdot \underline{y}+\underline{x} \wedge \underline{y}$,

with

$\underline{x} \cdot \underline{y}:=-\langle\underline{x}, \underline{y}\rangle=-\sum_{j=1}^{m} x_{j} y_{j}=\frac{1}{2}(\underline{x} \underline{y}+\underline{y} \underline{x})$

and

$\underline{x} \wedge \underline{y}:=\sum_{j<k} e_{j k}\left(x_{j} y_{k}-x_{k} y_{j}\right)=\frac{1}{2}(\underline{x} \underline{y}-\underline{y} \underline{x})$.

Note that the square of a vector variable $\underline{x}$ is scalarvalued and equals the norm squared up to a minus sign: $\underline{x}^{2}=-\langle\underline{x}, \underline{x}\rangle=-|\underline{x}|^{2}$.

We also introduce a first order vector differential operator by

$\partial_{\underline{x}}=\sum_{j=1}^{m} \partial_{x_{j}} e_{j}$.

This operator is the so-called Dirac operator. Its square equals, up to a minus sign, the Laplace operator in $\mathbb{R}^{m}$ : $\partial_{\underline{x}}^{2}=-\Delta$.
The Dirac operator $\partial_{\underline{x}}$ acts on functions $f$ taking values in $\mathcal{C l}_{0, m}$. Such functions can always be decomposed as

$$
\begin{aligned}
f(x)= & f_{0}(x)+\sum_{i=1}^{m} e_{i} f_{i}(x) \\
& +\sum_{i<j} e_{i} e_{j} f_{i j}(x)+\ldots+e_{1} \ldots e_{m} f_{1 \ldots m}(x)
\end{aligned}
$$

with $f_{0}, f_{i}, f_{i j}, \ldots, f_{1 \ldots m}$ all real-valued functions. As $f$ has in total $2^{m}$ components (which equals the dimension of $\mathcal{C} l_{0, m}$ ), this is the maximal number of components of the signals that can be studied using the transforms in the next section.

Finally, another important operator in Clifford analysis is the so-called Gamma operator, defined by

$\Gamma_{\underline{x}}=-\underline{x} \wedge \partial_{\underline{x}}=-\sum_{j<k} e_{j k}\left(x_{j} \partial_{x_{k}}-x_{k} \partial_{x_{j}}\right)$.

This operator is bivector-valued.

\section{Overview of recent results}

In the paper [13] we started our analysis from 4 different, yet equivalent, definitions of the classical Fourier transform in $\mathbb{R}^{m}$. Each of these formulations allows for generalization to hypercomplex FTs. We summarize them briefly. The first and most basic formulation is of course given by the integral transform

$\mathbf{F} 1 \quad \mathcal{F}(f)(\underline{y})=\frac{1}{(2 \pi)^{m / 2}} \int_{\mathbb{R}^{m}} e^{-i\langle\underline{x}, \underline{y}\rangle} f(\underline{x}) d x$

with $\langle\underline{x}, \underline{y}\rangle$ the standard inner product and $d x$ the Lebesgue measure on $\mathbb{R}^{m}$. Alternatively, one can rewrite the transform as

F2 $\mathcal{F}(f)(\underline{y})=\frac{1}{(2 \pi)^{m / 2}} \int_{\mathbb{R}^{m}} K(\underline{x}, \underline{y}) f(\underline{x}) d x$

where $K(\underline{x}, \underline{y})$ is, up to a multiplicative constant, the unique solution of the system of PDEs

$\partial_{y_{j}} K(\underline{x}, \underline{y})=-i x_{j} K(\underline{x}, \underline{y}), \quad j=1, \ldots, m$.

Yet another formulation is given by

F3 $\mathcal{F}=e^{\frac{i \pi m}{4}} e^{\frac{i \pi}{4}\left(\Delta-|\underline{x}|^{2}\right)}$

with $\Delta$ the Laplacian in $\mathbb{R}^{m}$. This expression connects the Fourier transform with the Lie algebra $\mathfrak{s l}_{2}$ generated by $\Delta$ and $|\underline{x}|^{2}$ and with the theory of the quantum 
harmonic oscillator. Finally, the kernel can also be expressed as an infinite series in terms of special functions as

$$
\text { F4 } \begin{aligned}
K(\underline{x}, \underline{y}) & =2^{\lambda} \Gamma(\lambda)(|\underline{x}||\underline{y}|)^{-\lambda} \sum_{k=0}^{\infty}(k+\lambda)(-i)^{k} \\
& \times J_{k+\lambda}(|\underline{x}||\underline{y}|) C_{k}^{\lambda}(\langle\underline{\xi}, \underline{\eta}\rangle),
\end{aligned}
$$

where $\underline{\xi}=\underline{x} /|\underline{x}|, \underline{\eta}=\underline{y} /|\underline{y}|$ and $\lambda=(m-2) / 2$. Here, $J_{\nu}$ is the Bessel function and $C_{k}^{\lambda}$ the Gegenbauer polynomial.

Each formulation has its specific advantages and uses. F1 immediately yields a bound of the kernel and is hence ideal to study the transform on $L_{1}$ spaces or more general function spaces. F2 gives the calculus properties of the transform. F3 emphasizes the structural (Lie algebraic) properties of the Fourier transform and allows to compute its eigenfunctions and spectrum. Finally, F4 connects the Fourier transform with the theory of special functions, and is the ideal formulation to obtain e.g. the Bochner identities.

These 4 definitions serve as guidance in defining hypercomplex Fourier transforms, as each definition gives access to a crucial piece of information about the transform. A suitable hypercomplex transform should hence be expressible in these 4 different ways.

So far, we have applied these ideas in 3 different directions of hypercomplex FTs, namely

- $k$-vector Fourier transforms ([14])

- radially deformed Fourier transforms $([15,16])$

- Clifford-Fourier transforms $([17,13])$.

We briefly discuss the first 2 directions, after which we focus on the Clifford-Fourier transform. $k$-vector Fourier transforms are transforms with integral kernel $K(\underline{x}, \underline{y})$ a $k$-vector valued function (i.e. taking values in $\left.\mathcal{C} l_{0, m}^{k}\right)$ which is moreover a solution to the system of PDEs (compare with F2)

$\partial_{\underline{y}} K(\underline{x}, \underline{y})=-i K(\underline{x}, \underline{y}) \underline{x}$.

Contrary to the classical case, the system (2) does not have a single unique solution but instead a whole family of suitable solutions.

Radially deformed Fourier transforms are obtained by adapting the formulation F3 of the classical Fourier transform to

$\mathcal{F}_{\mathbf{D}}=e^{i \frac{\pi}{2}\left(\frac{1}{2}+\frac{m-1}{2(1+c)}\right)} e^{\frac{-i \pi}{4(1+c)^{2}}\left(\mathbf{D}^{2}-(1+c)^{2} \underline{x}^{2}\right)}$.

with

$\mathbf{D}=\partial_{\underline{x}}+c|\underline{x}|^{-2} \underline{x} \sum_{j=1}^{m} x_{j} \partial_{x_{j}}$, a radial deformation (depending on the numerical parameter $c \in \mathbb{R}$ ) of the Dirac operator $\partial_{\underline{x}}$ underlying the Laplace operator. So far, for this class of transforms also formulation $\mathbf{F 2}$ and $\mathbf{F} 4$ have been obtained, but the question to find an explicit analytical expression for its kernel (formulation F1) is extremely hard.

The situation is quite different for the Clifford-Fourier transform (CFT), where by now an almost complete treatment has been obtained. This transform was first introduced in [18] and further studied in [19,20]. It was initially also defined using a generalization of F3, namely

$\mathcal{F}_{ \pm}=e^{\frac{i \pi m}{4}} e^{\mp \frac{i \pi}{2} \Gamma} e^{\frac{i \pi}{4}\left(\Delta-|\underline{x}|^{2}\right)}=e^{\frac{i \pi m}{4}} e^{\frac{i \pi}{4}\left(\Delta-|\underline{x}|^{2} \mp 2 \Gamma\right)}$

with $\Gamma$ the Gamma operator. The motivation behind this definition was to find a couple of transforms $\mathcal{F}_{ \pm}$ such that

$\mathcal{F}_{+} \mathcal{F}_{-}=\mathcal{F}_{-} \mathcal{F}_{+}=\mathcal{F}^{2}$.

It turned out to be a difficult problem to find the other formulations for this particular transform. A breakthrough was recently obtained in [17]. As an analog of formulation $\mathbf{F} 4$ the following result was obtained:

Theorem 1 The Clifford-Fourier transform $\mathcal{F}_{-}=e^{\frac{i \pi m}{4}} e^{\frac{i \pi}{2} \Gamma} e^{\frac{i \pi}{4}\left(\Delta-|\underline{x}|^{2}\right)}$ is given by the integral transform

$\frac{1}{(2 \pi)^{m / 2}} \int_{\mathbb{R}^{m}} K_{-}(\underline{x}, \underline{y}) f(\underline{x}) d x$

with integral kernel

$K_{-}(\underline{x}, \underline{y})=A_{\lambda}+B_{\lambda}+(\underline{x} \wedge \underline{y}) C_{\lambda}$

with

$$
\begin{aligned}
A_{\lambda}(w, z)= & 2^{\lambda-1} \Gamma(\lambda+1) z^{-\lambda} \sum_{k=0}^{\infty}\left(i^{2 \lambda+2}+(-1)^{k}\right) \\
& \times J_{k+\lambda}(z) C_{k}^{\lambda}(w), \\
B_{\lambda}(w, z)= & -2^{\lambda-1} \Gamma(\lambda) z^{-\lambda} \sum_{k=0}^{\infty}(k+\lambda)\left(i^{2 \lambda+2}-(-1)^{k}\right) \\
& \times J_{k+\lambda}(z) C_{k}^{\lambda}(w), \\
C_{\lambda}(w, z)= & -2^{\lambda} \Gamma(\lambda+1) z^{-\lambda-1} \sum_{k=1}^{\infty}\left(i^{2 \lambda+2}+(-1)^{k}\right) \\
& \times J_{k+\lambda}(z) C_{k-1}^{\lambda+1}(w),
\end{aligned}
$$

where $z=|\underline{x}||\underline{y}|, w=\langle\underline{\xi}, \underline{\eta}\rangle$ and $\lambda=(m-2) / 2$.

Note that it can be computed that the kernel $K_{+}(\underline{x}, \underline{y})$ of $\mathcal{F}_{+}$is given by $K_{+}(\underline{x}, \underline{y})=\overline{K_{-}(\underline{x},-\underline{y})}$ where the bar denotes complex conjugation.

Moreover, it was then possible to obtain formulation $\mathbf{F 1}$ in the case where the dimension $m$ is even. 
Theorem 2 The kernel of the Clifford-Fourier transform in even dimension $m>2$ is given by

$$
\begin{aligned}
K_{-}(\underline{x}, \underline{y}) & =e^{i \frac{\pi}{2} \Gamma_{\underline{y}}} e^{-i\langle\underline{x}, \underline{y}\rangle} \\
& =(-1)^{\frac{m}{2}}\left(\frac{\pi}{2}\right)^{\frac{1}{2}}\left(A_{(m-2) / 2}^{*}(s, t)\right. \\
& \left.+B_{(m-2) / 2}^{*}(s, t)+(\underline{x} \wedge \underline{y}) C_{(m-2) / 2}^{*}(s, t)\right)
\end{aligned}
$$

where $s=\langle\underline{x}, \underline{y}\rangle$ and $t=|\underline{x} \wedge \underline{y}|=\sqrt{|\underline{x}|^{2}|\underline{y}|^{2}-s^{2}}$ and

$$
\begin{aligned}
A_{(m-2) / 2}^{*}(s, t) & =\sum_{\ell=0}^{\left\lfloor\frac{m}{4}-\frac{3}{4}\right\rfloor} s^{m / 2-2-2 \ell} \frac{1}{2^{\ell} \ell !} \\
& \times \frac{\Gamma\left(\frac{m}{2}\right)}{\Gamma\left(\frac{m}{2}-2 \ell-1\right)} \widetilde{J}_{(m-2 \ell-3) / 2}(t), \\
B_{(m-2) / 2}^{*}(s, t) & =-\sum_{\ell=0}^{\left\lfloor\frac{m}{4}-\frac{1}{2}\right\rfloor} s^{m / 2-1-2 \ell} \frac{1}{2^{\ell} \ell !} \\
& \times \frac{\Gamma\left(\frac{m}{2}\right)}{\Gamma\left(\frac{m}{2}-2 \ell\right)} \widetilde{J}_{(m-2 \ell-3) / 2}(t), \\
C_{(m-2) / 2}^{*}(s, t) & =-\sum_{\ell=0}^{\left\lfloor\frac{m}{4}-\frac{1}{2}\right\rfloor} s^{m / 2-1-2 \ell} \frac{1}{2^{\ell} \ell !} \\
& \times \frac{\Gamma\left(\frac{m}{2}\right)}{\Gamma\left(\frac{m}{2}-2 \ell\right)} \widetilde{J}_{(m-2 \ell-1) / 2}(t)
\end{aligned}
$$

with $\widetilde{J}_{\alpha}(t)=t^{-\alpha} J_{\alpha}(t)$.

Subsequently, in [13] we studied the formulation F2 for the CFT, which determines the kernel of this transform as a solution of the following system of PDEs:

$$
\begin{aligned}
\partial_{\underline{y}}\left[K_{+}(\underline{x}, \underline{y})\right] & =(-i)^{m} K_{-}(\underline{x}, \underline{y}) \underline{x} \\
{\left[K_{+}(\underline{x}, \underline{y})\right] \partial_{\underline{x}} } & =(-i)^{m} \underline{y} K_{-}(\underline{x}, \underline{y}) .
\end{aligned}
$$

Indeed, it is e.g. easy to check that the result of Theorem 2 satisfies (3). However, a new phenomenon appears here, as the solution given by Theorem 2 is not the unique solution of the system (contrary to the case of the ordinary Fourier transform or the Dunkl transform, see [21]).

To obtain more insight in this phenomenon, we determined all solutions of (3). In even dimension, these are of the form

$$
K_{-}(\underline{x}, \underline{y})=f(s, t)+(\underline{x} \wedge \underline{y}) g(s, t)
$$

with $s=\langle\underline{x}, \underline{y}\rangle, t=|\underline{x} \wedge \underline{y}|$ and $f$ and $g$ real-valued functions. In this way, we aimed to find an explanation for the precise form of the kernel given in Theorem 2.

As result, we obtained, as solutions of (3), a set of $m-1$ kernels

$K_{-, m}^{j}(\underline{x}, \underline{y}), \quad j=0, \ldots, m-2$ that can be organized in a diagram as follows

$$
m=2 \quad m=4 \quad m=6 \quad m=8
$$

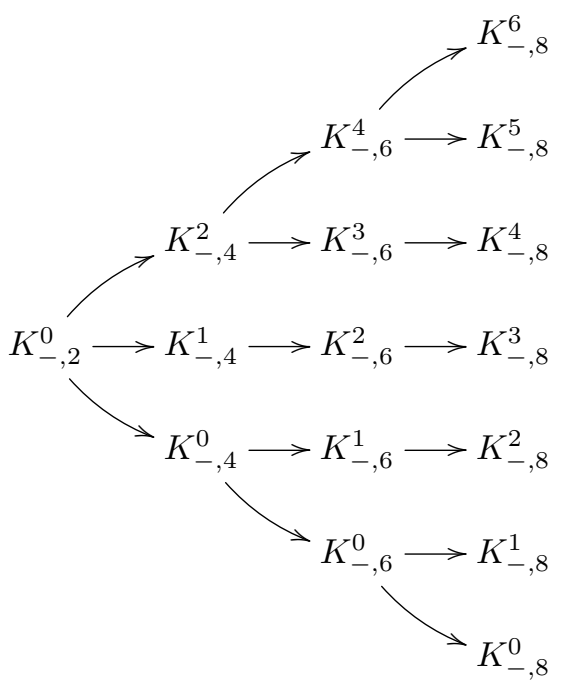

Here the arrows describe certain recursive relations (given explicitly in [13]).

The middle line, namely the kernels $K_{-, m}^{(m-2) / 2}(\underline{x}, \underline{y})$ correspond to the kernels obtained in Theorem 2. The other kernels yield new integral transforms that have the same calculus properties as the original CFT but with different spectrum. Of all these transforms, only the transform given by Theorem 2 is unitary. We observe that with each step in the dimension, two new kernels appear. In particular the kernels $K_{-, m}^{0}(\underline{x}, \underline{y})$ on the lower diagonal are interesting, as they give us the Fourier-Bessel transform which was previously introduced in [22] for different reasons.

For explicit formulas for the kernels $K_{-, m}^{j}(\underline{x}, y)$ we refer the reader to formula (4.6) and Theorem 4.2 (where series representations as in $\mathbf{F} 4$ are obtained) in [13].

Also in the odd dimensional case, we can solve the system (3). The result can again be organized in a scheme, similar to the one above in the even dimensional case. Although the Fourier-Bessel kernel (in odd dimension) again appears on the lower diagonal, the Clifford-Fourier kernel can no longer be found in this scheme. For details, we again refer to [13].

\section{Fractional version of the Clifford-Fourier transform}

In this section, we show how a fractional version of the CFT can be obtained. To that end, we adapt the definition F3

$\mathcal{F}_{ \pm}=e^{\frac{i \pi m}{4}} e^{\frac{i \pi}{4}\left(\Delta-|\underline{x}|^{2} \mp 2 \Gamma\right)}$ 
to

$\mathcal{F}_{ \pm, \alpha}=e^{\frac{i \alpha m}{2}} e^{\frac{i \alpha}{2}\left(\Delta-|\underline{x}|^{2} \mp 2 \Gamma\right)}$

where $\alpha \in]-\pi, \pi[$ and $\alpha \neq 0$. Notice that we have immediately

$\mathcal{F}_{+, \alpha} \mathcal{F}_{-, \alpha}=\mathcal{F}_{\alpha}^{2}$

with $\mathcal{F}_{\alpha}=e^{\frac{i \alpha m}{2}} e^{\frac{i \alpha}{2}\left(\Delta-|\underline{x}|^{2}\right)}$ the fractional version of the ordinary Fourier transform, given explicitly by (see [23])

$$
\begin{aligned}
\mathcal{F}_{\alpha}(f)(\underline{y}) & =\left(\pi\left(1-e^{-2 i \alpha}\right)\right)^{-m / 2} \\
& \times \int_{\mathbb{R}^{m}} e^{-i\langle\underline{x}, \underline{y}\rangle / \sin \alpha} e^{\frac{i}{2}(\cot \alpha)\left(|\underline{x}|^{2}+|\underline{y}|^{2}\right)} f(\underline{x}) d x .
\end{aligned}
$$

Our aim is to find an integral expression for $\mathcal{F}_{-, \alpha}$ :

$$
\begin{aligned}
\mathcal{F}_{-, \alpha}(f)(\underline{y}) & =\left(\pi\left(1-e^{-2 i \alpha}\right)\right)^{-m / 2} \\
& \times \int_{\mathbb{R}^{m}} K_{-, \alpha}(\underline{x}, \underline{y}) f(\underline{x}) d x .
\end{aligned}
$$

We compute formally

$$
\begin{aligned}
\mathcal{F}_{-, \alpha} & =e^{\frac{i \alpha m}{2}} e^{\frac{i \alpha}{2}\left(\Delta-|\underline{x}|^{2}+2 \Gamma\right)} \\
& =e^{\frac{i \alpha m}{2}} e^{i \alpha \Gamma} e^{\frac{i \alpha}{2}\left(\Delta-|\underline{x}|^{2}\right)} \\
& =\left(\pi\left(1-e^{-2 i \alpha}\right)\right)^{-m / 2} e^{i \alpha \Gamma_{\underline{y}}} \\
& \times \int_{\mathbb{R}^{m}} e^{-i\langle\underline{x}, \underline{y}\rangle / \sin \alpha} e^{\frac{i}{2}(\cot \alpha)\left(|\underline{x}|^{2}+|\underline{y}|^{2}\right)}(.) d x
\end{aligned}
$$

hence the kernel of the fractional CFT is given by

$$
\begin{aligned}
K_{-, \alpha}(\underline{x}, \underline{y}) & =e^{i \alpha \Gamma_{\underline{y}}}\left(e^{-i\langle\underline{x}, \underline{y}\rangle / \sin \alpha} e^{\frac{i}{2}(\cot \alpha)\left(|\underline{x}|^{2}+|\underline{y}|^{2}\right)}\right) \\
& =e^{\frac{i}{2}(\cot \alpha)\left(|\underline{\mid x}|^{2}+|\underline{y}|^{2}\right)} e^{i \alpha \Gamma_{\underline{y}}}\left(e^{-i\langle\underline{x}, \underline{y}\rangle / \sin \alpha}\right)
\end{aligned}
$$

where the last line follows because $\Gamma_{y}$ commutes with $|\underline{y}|$. Now using the series expansion (1), the term $e^{i \alpha \Gamma_{\underline{y}}}\left(e^{-i\langle\underline{x}, \underline{y}\rangle / \sin \alpha}\right)$ can be computed. Indeed, we find $e^{i \alpha \Gamma_{\underline{y}}}\left(e^{-i\langle\underline{x}, \underline{y}\rangle / \sin \alpha}\right)$$$
=e^{i \alpha \Gamma_{\underline{y}}} \Gamma(\lambda) \sum_{k=0}^{\infty} 2^{\lambda}(k+\lambda)(i \sin \alpha)^{-k}
$$$$
\times(|\underline{x}||\underline{y}|)^{k} \widetilde{J}_{k+\lambda}\left(\frac{|\underline{x}||\underline{y}|}{\sin \alpha}\right) C_{k}^{\lambda}(\langle\underline{\xi}, \underline{\eta}\rangle)
$$$$
=\Gamma(\lambda) \sum_{k=0}^{\infty} 2^{\lambda}(k+\lambda)(i \sin \alpha)^{-k}
$$$$
\times \widetilde{J}_{k+\lambda}\left(\frac{|\underline{x}||\underline{y}|}{\sin \alpha}\right) e^{i \alpha \Gamma_{\underline{y}}}\left((|\underline{x}||\underline{y}|)^{k} C_{k}^{\lambda}(\langle\underline{\xi}, \underline{\eta}\rangle)\right)
$$

so we have reduced the problem to calculating $e^{i \alpha \Gamma_{\underline{y}}}\left((|\underline{x}||\underline{y}|)^{k} C_{k}^{\lambda}(\langle\underline{\xi}, \underline{\eta}\rangle)\right)$. This can be done in a manner analogous to Lemma 3.1 in [17]. The result is given in the following lemma.
Lemma 1 One has

$$
\begin{aligned}
& e^{i \alpha \Gamma_{\underline{y}}}(|\underline{x}||\underline{y}|)^{k} C_{k}^{\lambda}(\langle\underline{\xi}, \underline{\eta}\rangle) \\
& =\frac{1}{2}\left(e^{i \alpha(k+m-2)}+e^{-i \alpha k}\right)(|\underline{x}||\underline{y}|)^{k} C_{k}^{\lambda}(\langle\underline{\xi}, \underline{\eta}\rangle) \\
& -\frac{\lambda}{2(k+\lambda)}\left(e^{i \alpha(k+m-2)}-e^{-i \alpha k}\right)(|\underline{x}||\underline{y}|)^{k} C_{k}^{\lambda}(\langle\underline{\xi}, \underline{\eta}\rangle) \\
& +\frac{\lambda}{k+\lambda} \underline{x} \wedge \underline{y}\left(e^{i \alpha(k+m-2)}-e^{-i \alpha k}\right)(|\underline{x}||\underline{y}|)^{k-1} C_{k-1}^{\lambda+1}(\langle\underline{\xi}, \underline{\eta}\rangle) .
\end{aligned}
$$

Using this lemma and the previous computation, we arrive at the following series representation for the kernel of the fractional CFT.

Theorem 3 The fractional Clifford-Fourier transform $\mathcal{F}_{-, \alpha}=e^{\frac{i \alpha m}{2}} e^{\frac{i \alpha}{2}\left(\Delta-|\underline{x}|^{2}+2 \Gamma\right)}$ is given explicitly by the integral transform

$$
\left(\pi\left(1-e^{-2 i \alpha}\right)\right)^{-m / 2} \int_{\mathbb{R}^{m}} K_{-, \alpha}(\underline{x}, \underline{y}) f(\underline{x}) d x
$$

with kernel

$K_{-, \alpha}(\underline{x}, \underline{y})=\left(A_{\lambda}+B_{\lambda}+(\underline{x} \wedge \underline{y}) C_{\lambda}\right) e^{\frac{i}{2}(\cot \alpha)\left(|\underline{x}|^{2}+|\underline{y}|^{2}\right)}$

with

$$
\begin{aligned}
A_{\lambda}(w, \widetilde{z})= & -2^{\lambda-1} \Gamma(\lambda+1) \widetilde{z}^{-\lambda} \sum_{k=0}^{\infty} i^{-k}\left(e^{i \alpha(k+2 \lambda)}-e^{-i \alpha k}\right) \\
& \times J_{k+\lambda}(\widetilde{z}) C_{k}^{\lambda}(w), \\
B_{\lambda}(w, \widetilde{z})= & 2^{\lambda-1} \Gamma(\lambda) \widetilde{z}^{-\lambda} \sum_{k=0}^{\infty}(k+\lambda) i^{-k}\left(e^{i \alpha(k+2 \lambda)}+e^{-i \alpha k}\right) \\
& \times J_{k+\lambda}(\widetilde{z}) C_{k}^{\lambda}(w), \\
C_{\lambda}(w, \widetilde{z})= & \frac{2^{\lambda} \Gamma(\lambda+1)}{\sin \alpha} \widetilde{z}^{-\lambda-1} \sum_{k=1}^{\infty} i^{-k}\left(e^{i \alpha(k+2 \lambda)}-e^{-i \alpha k}\right) \\
& \times J_{k+\lambda}(\widetilde{z}) C_{k-1}^{\lambda+1}(w)
\end{aligned}
$$

where $\widetilde{z}=(|\underline{x}||\underline{y}|) / \sin \alpha, w=\langle\xi, \underline{\eta}\rangle$ and $\lambda=(m-2) / 2$.

In a similar way, one can obtain a series expansion for the kernel $K_{+, \alpha}(\underline{x}, \underline{y})$.

Remark 1 A different type of a fractional hypercomplex FT is studied in [24]. This transform is only defined over the quaternions and not over a general Clifford algebra.

\section{Kernel of the fractional CFT in dimension 2}

In this section, we give an explicit construction of the kernel $K_{-, \alpha}(\underline{x}, \underline{y})$ of the fractional CFT in dimension 2 (resulting in Theorem 4). 
The method we use is an adaptation of the one designed in [19]. In this method, the following Clifford numbers play a crucial role:

$P^{ \pm}=\frac{1}{2}\left(1 \pm i e_{12}\right)$

They are self-adjoint mutually orthogonal idempotents which, by multiplication, transform $e_{12}$ into the imaginary unit $i$. Hence, they satisfy the following properties: (i)

$P^{+}+P^{-}=1 ; \quad P^{+} P^{-}=P^{-} P^{+}=0 ;$

$\left(P^{ \pm}\right)^{2}=P^{ \pm}$

(ii) for $k \in \mathbb{N}$ :

$P^{+}\left(i e_{12}\right)^{k}=P^{+} \quad$ or $\quad P^{+}\left(e_{12}\right)^{k}=P^{+}(-i)^{k}$;

$P^{-}\left(i e_{12}\right)^{k}=(-1)^{k} P^{-}$or $\quad P^{-}\left(e_{12}\right)^{k}=P^{-} i^{k}$.

Proof See [20], Lemma 4.4, p. 123.

By means of $P^{+}+P^{-}=1$ and $\Gamma_{y}=-e_{12} L_{12}$ with $L_{12}=y_{1} \partial_{y_{2}}-y_{2} \partial_{y_{1}}$ the Euler angle, we now compute that

$$
\begin{aligned}
e^{i \alpha \Gamma_{\underline{y}}}\left(e^{-i\langle\underline{x}, \underline{y}\rangle / \sin \alpha}\right) & =P^{+} e^{-i \alpha e_{12} L_{12}}\left(e^{-i\langle\underline{x}, \underline{y}\rangle / \sin \alpha}\right) \\
& +P^{-} e^{-i \alpha e_{12} L_{12}}\left(e^{-i\langle\underline{x}, \underline{y}\rangle / \sin \alpha}\right) .
\end{aligned}
$$

Moreover, using property (4), we obtain

$$
\begin{aligned}
P^{+} e^{-i \alpha e_{12} L_{12}} & =P^{+} \sum_{k=0}^{\infty} \frac{\left(-i e_{12}\right)^{k}}{k !}\left(\alpha L_{12}\right)^{k} \\
& =P^{+} \sum_{k=0}^{\infty} \frac{(-1)^{k}}{k !}\left(\alpha L_{12}\right)^{k} \\
& =P^{+} e^{-\alpha L_{12}}
\end{aligned}
$$

and similarly, using (5), we find

$P^{-} e^{-i \alpha e_{12} L_{12}}=P^{-} e^{\alpha L_{12}}$.

Hence, equation (6) becomes

$$
\begin{aligned}
e^{i \alpha \Gamma_{\underline{y}}}\left(e^{-i\langle\underline{x}, \underline{y}\rangle / \sin \alpha}\right) & =P^{+} e^{-\alpha L_{12}}\left(e^{-i\langle\underline{x}, \underline{y}\rangle / \sin \alpha}\right) \\
& +P^{-} e^{\alpha L_{12}}\left(e^{-i\langle\underline{x}, \underline{y}\rangle / \sin \alpha}\right) .
\end{aligned}
$$

We now prove the following intermediate result.

Lemma 2 Let $f$ be a real-analytic function in $\mathbb{R}^{2}$, let $L_{12}=y_{1} \partial_{y_{2}}-y_{2} \partial_{y_{1}}$ be the Euler angle, $\left.\alpha \in\right]-\pi, \pi[$ and let $R_{\alpha}^{ \pm}$be the operator exponential given by

$R_{\alpha}^{ \pm}:=e^{ \pm \alpha L_{12}}$.

\section{Then one has}

$R_{\alpha}^{ \pm}\left[f\left(y_{1}, y_{2}\right)\right]=f\left(\cos \alpha y_{1} \mp \sin \alpha y_{2}, \pm \sin \alpha y_{1}+\cos \alpha y_{2}\right)$.
Proof In terms of polar coordinates

$\left\{\begin{array}{l}y_{1}=r \cos \theta \\ y_{2}=r \sin \theta\end{array}\right.$

with $r=|\underline{y}| \in[0,+\infty[$ and $\theta \in[0,2 \pi[$, the operator exponential $R_{\alpha}^{+}$takes the form

$R_{\alpha, \theta}^{+}:=e^{\alpha \partial_{\theta}}$

We have

$$
\begin{aligned}
R_{\alpha, \theta}^{+}[f(r, \theta)] & =R_{\alpha, \psi}^{+}[f(r, \theta+\psi)]_{\psi=0} \\
& =\sum_{k=0}^{\infty} \frac{1}{k !} \alpha^{k} \partial_{\psi}^{k}[f(r, \theta+\psi)]_{\psi=0} .
\end{aligned}
$$

As we assume $f$ to be real-analytic in $\mathbb{R}^{2}$, this becomes $R_{\alpha, \theta}^{+}[f(r, \theta)]=f(r, \theta+\alpha)$,

which leads to the desired result. The result for the operator exponential $R_{\alpha}^{-}$is proved in a similar way.

Applying the above lemma to equation (7) yields:

$$
\begin{aligned}
& e^{i \alpha \Gamma_{\underline{y}}}\left(e^{-i\langle\underline{x}, \underline{y}\rangle / \sin \alpha}\right) \\
& =P^{+} e^{-\frac{i}{\sin \alpha}\left(x_{1} y_{1} \cos \alpha+x_{1} y_{2} \sin \alpha-x_{2} y_{1} \sin \alpha+x_{2} y_{2} \cos \alpha\right)} \\
& +P^{-} e^{-\frac{i}{\sin \alpha}\left(x_{1} y_{1} \cos \alpha-x_{1} y_{2} \sin \alpha+x_{2} y_{1} \sin \alpha+x_{2} y_{2} \cos \alpha\right)} \\
& =P^{+} e^{-i\left(x_{1} y_{2}-x_{2} y_{1}\right)} e^{-i \cot \alpha\langle\underline{x}, \underline{y}\rangle} \\
& +P^{-} e^{-i\left(x_{2} y_{1}-x_{1} y_{2}\right)} e^{-i \cot \alpha\langle\underline{x}, \underline{y}\rangle} .
\end{aligned}
$$

Next, by means of property (4), we have consecutively

$$
\begin{aligned}
P^{+} e^{-i\left(x_{1} y_{2}-x_{2} y_{1}\right)} & =P^{+} \sum_{k=0}^{\infty} \frac{(-i)^{k}}{k !}\left(x_{1} y_{2}-x_{2} y_{1}\right)^{k} \\
& =P^{+} \sum_{k=0}^{\infty} \frac{\left(e_{12}\right)^{k}}{k !}\left(x_{1} y_{2}-x_{2} y_{1}\right)^{k} \\
& =P^{+} e^{(\underline{x} \wedge \underline{y})}
\end{aligned}
$$

because in dimension two one has $\underline{x} \wedge y:=e_{12}\left(x_{1} y_{2}-x_{2} y_{1}\right)$. Similary, applying (5) we find

$P^{-} e^{-i\left(x_{2} y_{1}-x_{1} y_{2}\right)}=P^{-} e^{(\underline{x} \wedge \underline{y})}$.

Hence, we arrive at

$$
\begin{aligned}
e^{i \alpha \Gamma_{\underline{y}}}\left(e^{-i\langle\underline{x}, \underline{y}\rangle / \sin \alpha}\right) & =\left(P^{+}+P^{-}\right) e^{(\underline{x} \wedge \underline{y})} e^{-i \cot \alpha\langle\underline{x}, \underline{y}\rangle} \\
& =e^{(\underline{x} \wedge \underline{y})} e^{-i \cot \alpha\langle\underline{x}, \underline{y}\rangle} .
\end{aligned}
$$


Finally, taking into account that

$$
\begin{aligned}
e^{(\underline{x} \wedge \underline{y})} & =\sum_{k=0}^{\infty} \frac{1}{2 k !}\left(-|\underline{x} \wedge \underline{y}|^{2}\right)^{k} \\
& +(\underline{x} \wedge \underline{y}) \sum_{k=0}^{\infty} \frac{1}{(2 k+1) !}\left(-|\underline{x} \wedge \underline{y}|^{2}\right)^{k} \\
& =\sum_{k=0}^{\infty} \frac{(-1)^{k}}{2 k !} t^{2 k}+\frac{(\underline{x} \wedge \underline{y})}{t} \sum_{k=0}^{\infty} \frac{(-1)^{k}}{(2 k+1) !} t^{2 k+1} \\
& =\cos t+\frac{(\underline{x} \wedge \underline{y})}{t} \sin t,
\end{aligned}
$$

with $t=|\underline{x} \wedge \underline{y}|=\sqrt{|\underline{x}|^{2}|\underline{y}|^{2}-\langle\underline{x}, \underline{y}\rangle^{2}}=\left|x_{1} y_{2}-x_{2} y_{1}\right|$, we can summarize our result in the following Theorem.

Theorem 4 (Fractional Clifford-Fourier kernel, $m=2$ ) The kernel of the fractional Clifford-Fourier transform is given by

$$
\begin{aligned}
K_{-, \alpha}(\underline{x}, \underline{y}) & =e^{\frac{i}{2}(\cot \alpha)\left(|\underline{x}|^{2}+|\underline{y}|^{2}\right)} e^{i \alpha \Gamma_{\underline{y}}}\left(e^{-i\langle\underline{x}, \underline{y}\rangle / \sin \alpha}\right) \\
& =\left(\cos t+(\underline{x} \wedge \underline{y}) \frac{\sin t}{t}\right) \\
& \times e^{-i\langle\underline{x}, \underline{y}\rangle \cot \alpha} e^{\frac{i}{2}(\cot \alpha)\left(|\underline{x}|^{2}+|\underline{y}|^{2}\right)}
\end{aligned}
$$

with $t=|\underline{x} \wedge \underline{y}|=\sqrt{|\underline{x}|^{2}|\underline{y}|^{2}-\langle\underline{x}, \underline{y}\rangle^{2}}$.

Let us now discuss some properties of the two-dimensional fractional CFT. First we note that the kernel can be written explicitly as

$$
\begin{aligned}
K_{-, \alpha}(\underline{x}, \underline{y}) & =\left(\cos t+e_{1} e_{2}\left(x_{1} y_{2}-x_{2} y_{1}\right) \frac{\sin t}{t}\right) \\
& \times e^{-i\langle\underline{x}, \underline{y}\rangle \cot \alpha} e^{\frac{i}{2}(\cot \alpha)\left(|\underline{x}|^{2}+|\underline{y}|^{2}\right)} .
\end{aligned}
$$

Both the scalar component and the component of $e_{1} e_{2}$ are clearly bounded, as

$\left|\left(x_{1} y_{2}-x_{2} y_{1}\right) \frac{\sin t}{t}\right| \leq|\sin t| \leq 1$.

Hence the resulting integral transform

$\left(\pi\left(1-e^{-2 i \alpha}\right)\right)^{-1} \int_{\mathbb{R}^{2}} K_{-, \alpha}(\underline{x}, \underline{y}) f(\underline{x}) d x$

is well-defined on $L_{1}\left(\mathbb{R}^{2}\right) \otimes \mathcal{C} l_{0,2}$, the space of $L_{1}$ functions taking values in the Clifford algebra associated with $\mathbb{R}^{2}$.

If one computes the kernel $K_{+, \alpha}(\underline{x}, \underline{y})$, one finds

$$
\begin{aligned}
K_{+, \alpha}(\underline{x}, \underline{y}) & =\left(\cos t-(\underline{x} \wedge \underline{y}) \frac{\sin t}{t}\right) \\
& \times e^{-i\langle\underline{x}, \underline{y}\rangle \cot \alpha} e^{\frac{i}{2}(\cot \alpha)\left(|\underline{x}|^{2}+|\underline{y}|^{2}\right)} .
\end{aligned}
$$

Both transforms $\mathcal{F}_{ \pm, \alpha}$ now satisfy nice calculus properties. They are given in the following theorem.
Theorem 5 Let $f \in \mathcal{S}\left(\mathbb{R}^{2}\right) \otimes \mathcal{C} l_{0,2}$. Then

$$
\begin{aligned}
\mathcal{F}_{+, \alpha}\left(\left(\underline{x} \cos \alpha-i \sin \alpha \partial_{\underline{x}}\right) f\right) & =e^{-i \alpha} \underline{y} \mathcal{F}_{-, \alpha}(f), \\
\mathcal{F}_{+, \alpha}\left(\left(\cos \alpha \partial_{\underline{x}}-i \underline{x} \sin \alpha\right) f\right) & =e^{-i \alpha} \underline{\partial}_{\underline{y}} \mathcal{F}_{-, \alpha}(f) .
\end{aligned}
$$

Proof Using the explicit expressions for the kernels $K_{ \pm, \alpha}(\underline{x}, \underline{y})$ we can compute that the following identities hold:

$$
\begin{aligned}
e^{-i \alpha} \underline{y} K_{-, \alpha}(\underline{x}, \underline{y}) & =K_{+, \alpha}(\underline{x}, \underline{y})\left(i \sin \alpha \partial_{\underline{x}}+\cos \alpha \underline{x}\right), \\
e^{-i \alpha} \partial_{\underline{y}} K_{-, \alpha}(\underline{x}, \underline{y}) & =-K_{+, \alpha}(\underline{x}, \underline{y})\left(i \sin \alpha \underline{x}+\cos \alpha \partial_{\underline{x}}\right) .
\end{aligned}
$$

In these formulas, the Dirac operator $\partial_{\underline{x}}$ is acting from the right on $K_{+, \alpha}$ as

$$
\left(K_{+, \alpha}\right) \partial_{\underline{x}}:=\sum_{j=1}^{2}\left(\partial_{x_{j}} K_{+, \alpha}\right) e_{j} .
$$

Because the kernel is bounded, we can now apply integration by parts. This yields the formulas stated in the theorem.

Finding an explicit analytic expression (as in F1) for the kernel of the fractional CFT in all dimensions is again a difficult problem. Hence we postpone its determination to a subsequent publication.

\section{Conclusion}

In this paper we have given an overview of a new approach in the design of hypercomplex FTs. We have put special emphasis on the Clifford-Fourier transform, as the canonical example in this line of research.

Moreover, we have defined a fractional version of the CFT and have determined a series expansion for its integral kernel. In the special case of dimension 2, we have even obtained explicit formulas and we were able to prove the calculus properties of the transform.

In future publications, we aim to study the fractional CFT in higher dimensions. Also the question of how to define convolution and translation for this new transform will be tackled.

\section{References}

1. Bülow, T. and Sommer, G.: Hypercomplex signals-a novel extension of the analytic signal to the multidimensional case. IEEE Trans. Signal Process. 49 (2001), 28442852.

2. Ebling, J. and Scheuermann, G.: Clifford Fourier transform on vector fields. IEEE Transactions on Visualization and Computer Graphics 11 (2005), 469-479.

3. Ell, T. and Sangwine, S.: Hypercomplex Fourier transforms of color images. IEEE Trans. Image Process. 16 (2007), 22-35. 
4. Moxey, C., Sangwine, S. and Ell, T.: Hypercomplex correlation techniques for vector images. IEEE Trans. Signal Process. 51 (2003), 1941-1953.

5. Said, S., Le Bihan, N. and Sangwine, S.: Fast complexified quaternion Fourier transform. IEEE Trans. Signal Process. 56 (2008), 1522-1531.

6. Kou, K. and Qian, T.: The Paley-Wiener theorem in $\mathbf{R}^{\mathbf{n}}$ with the Clifford analysis setting. J. Funct. Anal. 189 (2002), 227-241.

7. Kou, K. and Qian, T.: Shannon sampling in the Clifford analysis setting. Z. Anal. Anwendungen 24 (2005), 853870.

8. Li, C., McIntosh, A. and Qian, T.: Clifford algebras, Fourier transforms and singular convolution operators on Lipschitz surfaces. Rev. Mat. Iberoamericana 10 (1994), 665-721.

9. Brackx, F., Delanghe, R. and Sommen, F.: Clifford analysis, Research Notes in Mathematics, vol. 76. Pitman (Advanced Publishing Program), Boston, MA, 1982.

10. Delanghe, R., Sommen, F. and Souček, V.: Clifford algebra and spinor-valued functions, vol. 53 of Mathematics and its Applications. Kluwer Academic Publishers Group, Dordrecht, 1992.

11. Yuan, L., Yu, Z., Chen, S., Luo, W., Wang, Y. and Lü, G.: CAUSTA: Clifford algebra-based unified spatio-temporal analysis. Transactions in GIS 14 (2010), 59-83.

12. Bujack, R., Scheuermann, G. and Hitzer, E.: A general geometric Fourier transform. In 9th International Conference on Clifford Algebras and their Applications in Mathematical Physics. K. Gürlebeck (ed.). Weimar, Germany, 15-20 July 2011. 19 pages

13. De Bie, H., De Schepper, N. and Sommen, F.: The class of Clifford-Fourier transforms. Accepted for publication in J. Fourier Anal. Appl., arXiv:1101.1793, 30 pages.

14. De Bie, H. and Sommen, F.: Vector and bivector Fourier transforms in Clifford analysis. In 18th International Conference on the Application of Computer Science and Mathematics in Architecture and Civil Engineering. K. Gurlebeck and C. Könke (eds.), Weimar, Germany, 07-09 July 2009, 11 pages. Available online at 'http://euklid.bauing.uniweimar.de/ikm2009/paper.php'.

15. De Bie, H., Ørsted, B., Somberg, P. and Souček, V.: Dunkl operators and a family of realizations of $\mathfrak{o s p}(1 \mid 2)$. Accepted for publication in Trans. Amer. Math. Soc., arXiv:0911.4725, 28 pages.

16. De Bie, H., Ørsted, B., Somberg, P. and Souček, V.: The Clifford deformation of the Hermite semigroup. arXiv:1101.5551, 27 pages.

17. De Bie, H. and Xu, Y.: On the Clifford-Fourier transform. Int. Math. Res. Not. IMRN, (2011), 41 pages. doi: 10.1093/imrn/rnq288.

18. Brackx, F., De Schepper, N. and Sommen, F.: The Clifford-Fourier transform. J. Fourier Anal. Appl. 11 (2005), 669-681.

19. Brackx, F., De Schepper, N. and Sommen, F.: The twodimensional Clifford-Fourier transform. J. Math. Imaging Vision 26 (2006), 5-18.

20. Brackx, F., De Schepper, N. and Sommen, F.: The Fourier transform in Clifford analysis. Advances in Imaging and Electron Physics 156 (2008), 55-201.

21. de Jeu, M.F.E.: The Dunkl transform. Invent. Math. 113 (1993), 147-162.

22. Brackx, F., De Schepper, N. and Sommen, F.: The Fourier-Bessel transform. In 18th International Conference on the Application of Computer Science and Mathematics in Architecture and Civil Engineering. K Gurlebeck and C. Könke (eds.), Weimar, Germany, 07-09 July 2009, 18 pages. Available online at 'http://euklid.bauing.uniweimar.de/ikm2009/paper.php'.

23. Ozaktas, H., Zalevsky, Z. and Kutay, M.: The fractional Fourier transform. Wiley, Chichester, 2001.

24. Guanlei, X., Xiaotong, W. and Xiaogoang, X.: Fractional quaternionic Fourier transform, convolution and correlation. Signal Processing 88 (2008), 2511-2517. 